\title{
Reduction of Geomagnetic Effects (Periods T < 940 s) from Geoelectric Potential Difference Data
}

\author{
Makoto Harada ${ }^{*} \quad$ Non-member \\ Katsumi Hattori $^{* *}$ Member \\ Nobuhiro Isezaki ${ }^{* * *}$ Non-member
}

\begin{abstract}
An effective method is developed to identify and eliminate signals originated from external magnetic variations in the frequency range of 0.001 to $1 \mathrm{~Hz}$ in the geoelectric potential data. It is based on the interstation transfer function (ISTF) approach with the use of wavelet transform. Good performance has been found for the data at Kiyosumi station using Kakioka station as a reference, which means that almost whole of the inductive field could be eliminated successfully and other signals could be recognized with keeping their waveform. The detectability of simulated seismic electric signals (SES) has also been examined, and the result shows the successful discrimination of SES. These experimental facts indicate the effectiveness of our proposed signal reduction method.
\end{abstract}

Keywords : geoelectric potential data, external magnetic variations, interstation transfer function (ISTF), wavelet transform, seismic electric signals (SES)

\section{Introduction}

Electromagnetic phenomena preceding large earthquakes have been reported in various frequency ranges and they are considered as candidates for the short-term prediction ${ }^{(1)}$. ULF electromagnetc phenomena are most promising among them because of the sufficient skin depth ${ }^{(2) \sim(5)}$. The precursory geoelectric potential changes, called "Seismic Electric Signals (SES)", have been reported in Greece by the VAN group ${ }^{(6)(7)}$. In order to verify earthquake-related electromagnetic phenomena and clarify the possible physical mechanism(s), a network observation has been established in Japan ${ }^{(8) \sim(10)}$. The observed ULF magnetic and electric potential data are superposition of signals. The first one is originated from the external source field associated with the solar-terrestrial interactions such as the geomagnetic pulsations and geomagnetic storms, and their inductive field, which appears simultaneously in the global (hundreds of $\mathrm{km}$ ) scale. The socond one is the regional (a few tens of $\mathrm{km}$ ) signals such as artificial noises associated with the leakage current from DC-driven trains, and earthquake-related signals. The last one is local (less than few $\mathrm{kms}$ ) signals around the sensors. The signals associated with the crustal activity are very weak in general, and therefore the signal separation is of critical importance. As for the ULF geomagnetic data, we have already developed an effective method for elimination of the external source fields, which is based on the interstation transfer function (ISTF) method with wavelet transform. The performance of the designed filter is found to be quite good and geomagnetic pulsations are effectively reduced ${ }^{(11)}$.

In this paper, we extend the ISTF approach with wavelet

* Graduate School of Science and Technology, Chiba University 1-33, Yayoicho, Inage-ku, Chiba 263-8522

Now at Earthquake Prediction Research Center, Tokai University 3-20-1, Shimizu-Orido, Shizuoka 424-8610

** Marine Biosystems Research Center, Chiba University 1-33, Yayoicho, Inage-ku, Chiba 263-8522

*** Department of Earth Sciences, Faculty of Science, Chiba University 1-33, Yayoicho, Inage-ku, Chiba 263-8522

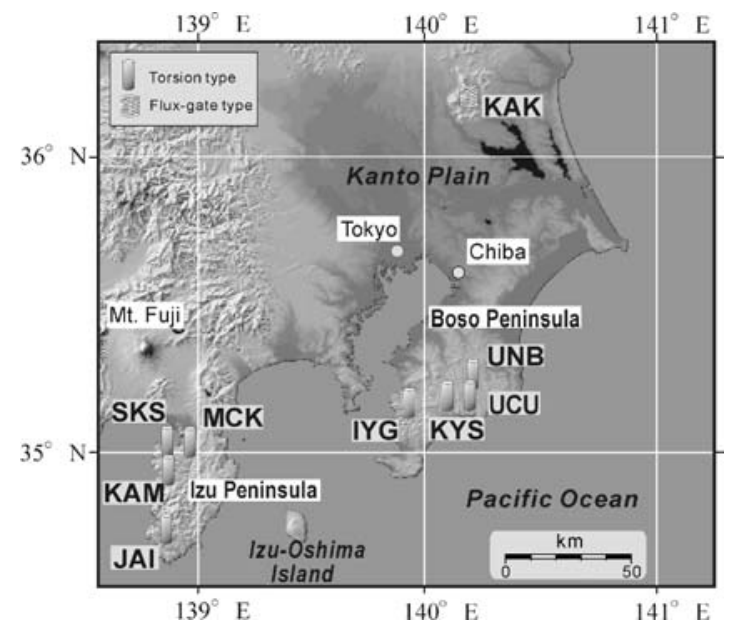

Fig. 1. The configuration map of geoelectric and magnetic stations in Kanto area, Japan (KAK indicates the Kakioka Magnetic Observatory, Japan Meteorological Agency. The geomagnetic data at KAK are used for remote reference)

transform to the geoelectric potential data with the aim of reducing the inductive fields due to geomagnetic field changes in the frequency range of 0.001 to $1 \mathrm{~Hz}$. Several articles have been devoted to reduce the inductive fields ${ }^{(12)(13)}$ in the electric potential data. They were based on the magnetotelluric approach ${ }^{(14)}$ with the conventional Fourier transform, using the simultaneous magnetic and electric data at the same site. Their results are effective for the elimination of inductive field. However, problems remain on the signal discrimination in the case of the appearance of correlative signals in the electric and magnetic data at the site. Therefore, the magnetic data observed at a remote reference station are useful because the earthquake-related signals are considered as regional. In this paper, we use geomagnetic data observed at Kakioka Magnetic Observatory, Japan Meteorological 
Agency, as the remote reference data. In order to evaluate the proposed method, we apply it to the electromagnetic data observed during a geomagnetic disturbance period. The detectability of simulated SESs is also examined.

\section{Formulation of Signal Discrimination}

In this section we show the formulation of ISTF approach for the reduction of inductive field from the geoelectric potential data.

2.1 Interstation Transfer Function (ISTF) The external source field in the frequency range of 0.001 to $0.2 \mathrm{~Hz}$ associated with the solar-terrestrial activities can be assumed as a plane wave which penetrates vertically to the earth ${ }^{(15)}$. Under this assumption, we can consider the observed three components of magnetic field on the ground $\mathbf{H}_{s}(\omega)=\left(\Delta X_{s}(\omega), \Delta Y_{s}(\omega), \Delta Z_{s}(\omega)\right)$ as the superposition of the following three inputs ${ }^{(16)(17)}$; (I) 'normal' field $\mathbf{H}_{n}(\omega)$ which is defined as the sum of the contributions from the external source field and the internal field due to regional horizontally layered electrical conductivity structure, (II) 'anomalous' field $\mathbf{H}_{a}(\omega)$ which is due to three-dimensional conductivity structure, and (III) other source fields $\delta \mathbf{H}(\omega)$. Then,

$$
\mathbf{H}_{s}(\omega)=\mathbf{H}_{n}(\omega)+\mathbf{H}_{a}(\omega)+\delta \mathbf{H}(\omega),
$$

where $\mathbf{H}_{n}(\omega)$ consists of the horizontal two components $\left(\Delta X_{n}(\omega)\right.$, $\left.\Delta Y_{n}(\omega), 0\right)$ because of the non-vertical component assumption of the source field. Since $\mathbf{H}_{a}(\omega)$ is considered as the secondary field of the input of $\mathbf{H}_{n}(\omega)$,

$$
\mathbf{H}_{a}(\omega)=\mathbf{T}(\omega) \cdot \mathbf{H}_{n}(\omega) .
$$

Substituting Eq.(2) to Eq.(1),

$$
\mathbf{H}_{s}(\omega)=\mathbf{T}^{\prime}(\omega) \cdot \mathbf{H}_{n}(\omega)+\delta \mathbf{H}(\omega),
$$

where $\mathbf{T}^{\prime}(\omega)=\mathbf{I}+\mathbf{T}(\omega)$ (I is a unit matrix). To get around some difficulties in solving Eq.(3) ${ }^{(18)}$, the remote reference data with sufficiently small artificial noise $\mathbf{H}_{r}(\omega)$ are adopted instead of $\mathbf{H}_{n}(\omega)$. Thus,

$$
\mathbf{H}_{s}(\omega)=\mathbf{T}_{i s}(\omega) \cdot \mathbf{H}_{r}(\omega)+\delta \mathbf{H}(\omega),
$$

When $\mathbf{H}_{r}(\omega)$ contains no artificial noise, it consists only of the horizontal two components $\left(\Delta X_{r}(\omega), \Delta Y_{r}(\omega), 0\right)$ by the assumption of the source field. $\mathbf{T}_{i s}(\omega)$ is the ISTF, $3 \times 2$ complex matrix. The ISTF should be estimated by using the data when the external source field is much greater than the uncorrelated part. We use the data during a severe geomagnetic storm at midnight time when the contribution of the artificial noises is small.

\subsection{Electromagnetic Transfer Function with ISTF} Approach It is well known that there exists a linear relationship between the observed electric and magnetic fields on the ground ${ }^{(14)}$.

$$
\mathbf{E}_{S}(\omega)=\mathbf{Z}_{S}(\omega) \cdot \mathbf{H}_{s}(\omega),
$$

where $\mathbf{E}_{s}(\omega)$ and $\mathbf{H}_{s}(\omega)$ indicate the horizontal two components of the observed electric and magnetic fields. $\mathbf{Z}_{s}(\omega)$ is a complex response function which is defined as a $2 \times 2$ matrix. Since $\mathbf{Z}_{s}(\omega)$ contains the information on the underground electrical structure, a large number of geophysical applications have been carried out so $\mathrm{far}^{(19)}$. Contamination of artificial noise prevents from the correct estimation of the transfer functions. Furthermore, if the desired signals such as earthquake-related ones appeared both in the electric and magnetic fields simultaneously, the signal discrimination may fail. The magnetic data should not contain any correlated signals with electric data except for the global geomagnetic changes. Accordingly, adopting the ISTF approach is essential to calculate the electromagnetic transfer function. The observed electric field is represented as follows;

$$
\mathbf{E}_{s}(\omega)=\mathbf{Z}_{s}(\omega) \cdot \mathbf{H}_{s}(\omega)+\delta \mathbf{E}(\omega)
$$

where $\mathbf{Z}_{s}(\omega) \cdot \mathbf{H}_{s}(\omega)$ indicates the inductive field and $\delta \mathbf{E}(\omega)$ represents the uncorrelated part, which is expected to contain the artificial noise and/or earthquake-related signals around the observation site. If sufficiently large external field is loaded, $\delta \mathbf{E}(\omega)$ could be neglegible. And in the study of magnetic ISTF, $\mathbf{T}_{i s}(\omega)$ is well estimated under the high multiple correlation value $\left(\gamma^{2}>0.9\right)$ between $\mathbf{H}_{s}(\omega)$ and $\mathbf{H}_{r}(\omega)^{(11)}$. Taking account of this condition and assuming ISTF to be time-invariant, Eq.(6) could be given as follows;

$$
\mathbf{E}_{s}(\omega)=\mathbf{Z}_{s}(\omega) \cdot \mathbf{T}_{i s}(\omega) \cdot \mathbf{H}_{r}(\omega)+\delta \mathbf{E}(\omega)
$$

Then, the relation between the electric field at a certain station $\mathbf{E}_{s}(\omega)$ and the magnetic field at the remote reference station $\mathbf{H}_{r}(\omega)$ is described by means of the interstation electromagnetic transfer function $\mathbf{Z}_{i s}(\omega)$;

$$
\begin{aligned}
& \mathbf{E}_{s}(\omega)=\mathbf{Z}_{i s}(\omega) \cdot \mathbf{H}_{r}(\omega)+\delta \mathbf{E}(\omega), \\
& \mathbf{Z}_{i s}(\omega)=\mathbf{Z}_{s}(\omega) \cdot \mathbf{T}_{i s}(\omega) \ldots \ldots \ldots \ldots \ldots
\end{aligned}
$$

2.3 Elimination of the Inductive Electric Field Related with External Source Field The estimated interstation electromagnetic transfer functions are time-invariant, because the electric property under the ground is supposedly stable when there is no large crustal changes. Taking acount of this, we can estimate the possible inductive field $\mathbf{E}_{s g}(\omega)$ at a certain station using the remote reference data.

$$
\mathbf{E}_{s g}(\omega)=\mathbf{Z}_{i s}(\omega) \cdot \mathbf{H}_{r}(\omega) \text {. }
$$

Therefore, if one subtracts the estimated inductive field from the observed data, it is expected to remove the global electromagnetic field changes.

$$
\Delta \mathbf{E}(\omega)=\mathbf{E}_{s}(\omega)-\mathbf{E}_{s g}(\omega),
$$

where $\Delta \mathbf{E}(\omega)$ indicates the residual component.

\subsection{Application of the Continuous Wavelet Transform}

The external source geomagnetic field is generally non-stationary, and the observed electric and magnetic data include many transient signals related with the DC-driven trains. In addition, the possible precursory signals of the geoelectric potential field, that is SES, often have a rectangular shape ${ }^{(6)(7)}$. We adopt here the wavelet transform ${ }^{(20)}$ to the above equations (Eqs. (1) $\sim(11)$ ) instead of the conventional Fourier transform. The mother wavelet is usually chosen depending on the signal properties and the purpose of analysis. In estimating the transfer functions from observed electric and magnetic data, the mother wavelet must be complex-valued and the relationship between scale and frequency should be relatively simple. The wavelet transform should characterize the amplitude and phase information of the electromagnetic fields both in the time and scale domains. These requirements are satisfied by the complex-valued Morlet wavelet ${ }^{(21)}$, consisting of a plane wave modulated by a Gaussian window;

$$
\psi_{0}(t)=\pi^{-1 / 4} e^{i \omega_{0} t} e^{-t^{2} / 2},
$$


(a) Magnetic field (upper line; KAK, lower line; KYS)

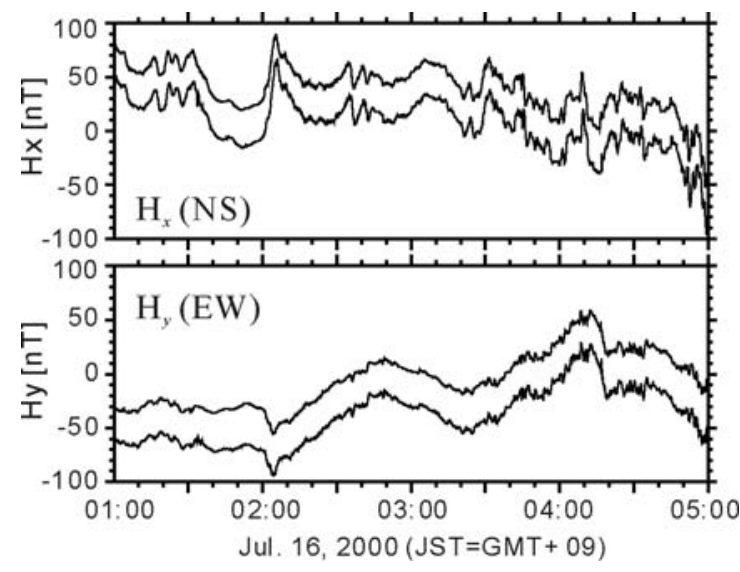

(b) Electric field at KYS (NS \& EW)

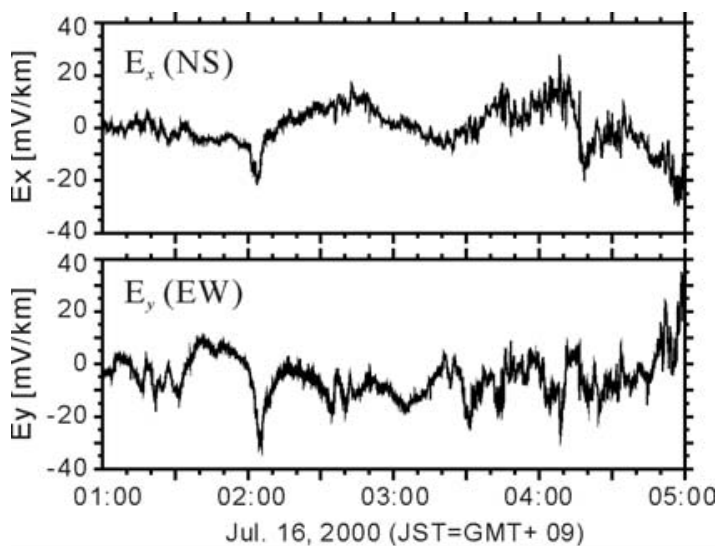

Fig. 2. The geomagnetic and geoelectric field variations in the period of 01:00 - 05:00 on July 16, 2000 (JST) (The geomagnetic activity was extremely high $(\mathrm{Kp}=9-))$; (a) The horizontal components of geomagnetic field at KAK (the upper line in each component) and KYS (the lower line) stations, (b) The horizontal components of electric field at KYS

where $t$ is a non-dimensional time parameter, and $\omega_{0}$ is a nondimensional frequency, here taken to be 6 to satisfy the admissibility condition. The relationship between Fourier and wavelet transforms has been studied ${ }^{(22)(23)}$, and the relationship between the wavelet scale, $a$, and the Fourier frequency, $\omega$, is given by ${ }^{(24)}$;

$$
a=\frac{\omega_{0}+\sqrt{2+\omega_{0}^{2}}}{2 \omega}
$$

The continuous wavelet transform of the real square integrable function $f(t) \in L^{2}(\mathfrak{R})$ at the location $b$, relative to a real integrable mother wavelet $\psi(t)$ at scale $a$, can be defined as;

$$
W f(b, a)=\left\langle f, \psi_{b, a}\right\rangle=\frac{1}{\sqrt{a}} \int_{-\infty}^{+\infty} f(t) \psi^{*}\left(\frac{t-b}{a}\right) d t,
$$

where $W f(b, a)$ is called the wavelet coefficient, * stands for the complex conjugate. In Fourier space, the wavelet transform can be expressed as;

$$
W f(b, a)=\frac{1}{2 \pi} \int_{-\infty}^{+\infty} \hat{f}(\omega) \hat{\psi}_{b, a}^{*}(\omega) e^{i b \omega} d \omega,
$$

where $\hat{f}$ and $\hat{\psi}$ are the Fourier transforms of $f$ and $\psi$,
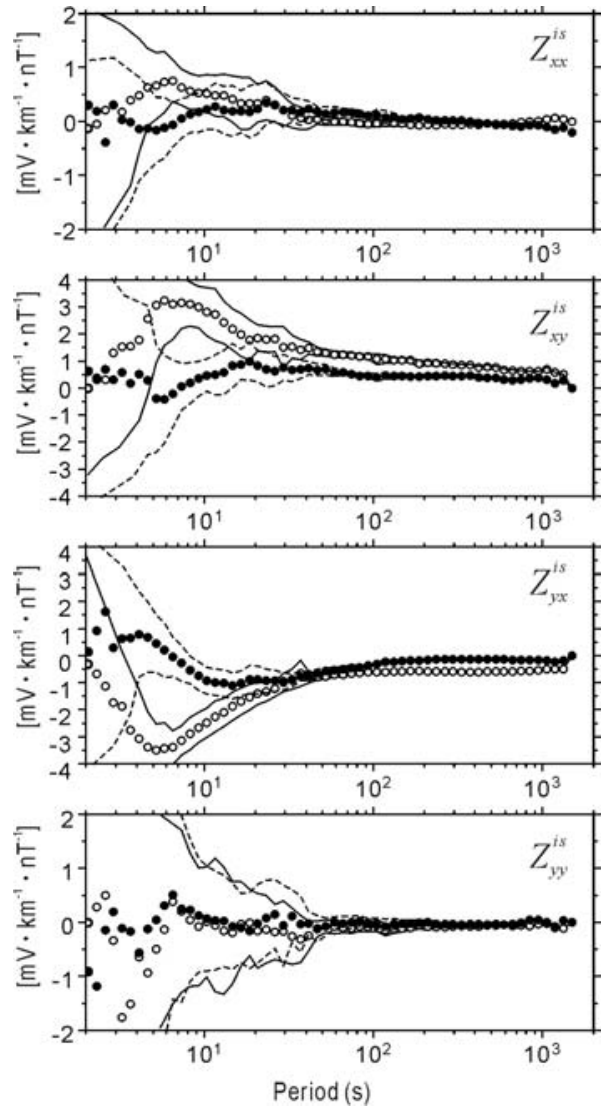

Fig. 3. The estimated interstation electromagnetic transfer functions $\mathbf{Z}_{i s}$ between $\mathbf{K A K}$ and KYS stations (Open and solid circles indicate the real and imaginary part of the transfer functions. The standard deviations are also shown)

respectively. The inverse wavelet transform is represented as follows;

$$
f(t)=\frac{1}{C_{\psi}} \cdot \frac{1}{a^{2}} \int_{b_{+}}^{+\infty} \int_{-\infty}^{+\infty} W f(b, a) \psi_{b, a}(t) d b d a
$$

where $C_{\psi}$ is the integral of $\|\hat{\psi}(\omega)\|^{2} \omega^{-1}$ over all frequencies.

Because of the redundancy in time and scale as for the continuous wavelet transform, we can reconstruct the time series using a completely different function from the mother wavelet ${ }^{(25)}$. We choose the $\delta$ function as the kernel for the reconstruction. In this case, the reconstructed time series is just the sum of the real part of the wavelet coefficients over all scales ${ }^{(25)}$.

$$
f(t)=\frac{d j d t^{1 / 2}}{C_{\delta} \psi_{0}(0)} \sum_{j=0}^{J} \frac{\mathfrak{R}\left\{W f\left(b, a_{j}\right)\right\}}{a_{j}^{1 / 2}}
$$

where $j$ indicates the scale index and $J$ yields the maximal number of resolvable scales. The factor $\psi_{0}(0)$ removes the energy scaling, while the $a_{j}^{1 / 2}$ converts the wavelet coefficient to an energy density. $\psi_{0}(0)$ and $\mathrm{C}_{\delta}$ are constant of $\pi^{-1 / 4}$ and 0.776 for the Morlet wavelet in this study, respectively ${ }^{(25)}$.

\section{Application to Observed Data}

In this section, the performance and capability of our proposed ISTF approach for reducing the inductive components will be examined by applying it to actual geoelectric potential difference data. Fig.1 shows the map of the configuration of our ULF 
(1) $E_{x}$ (NS)

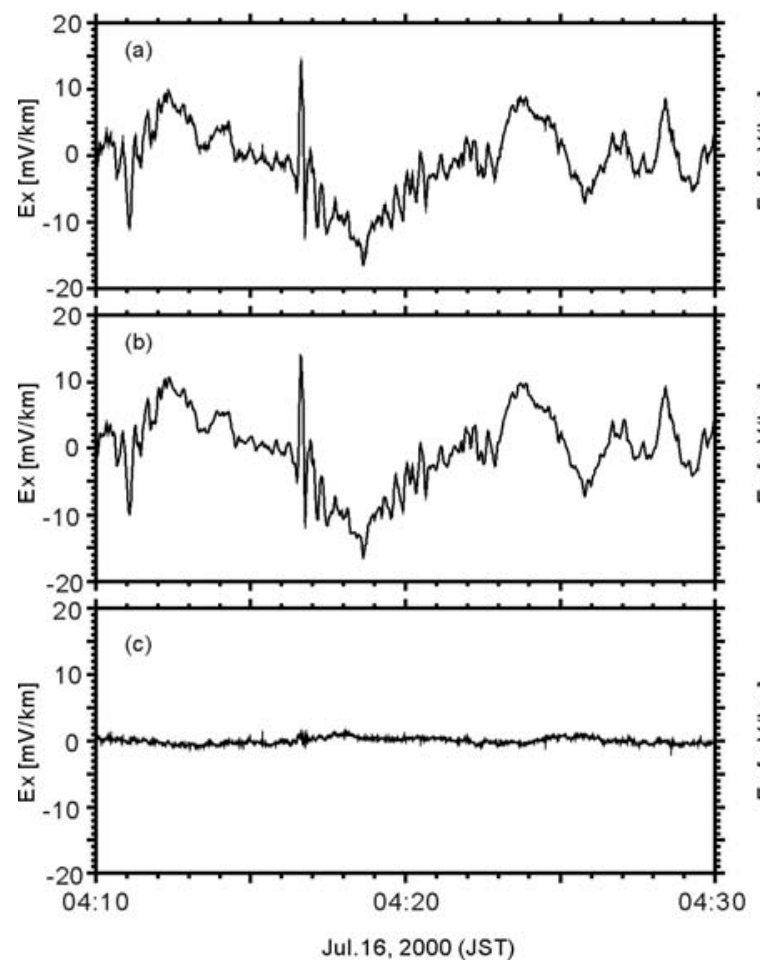

(2) $\mathrm{E}_{y}(\mathrm{EW})$

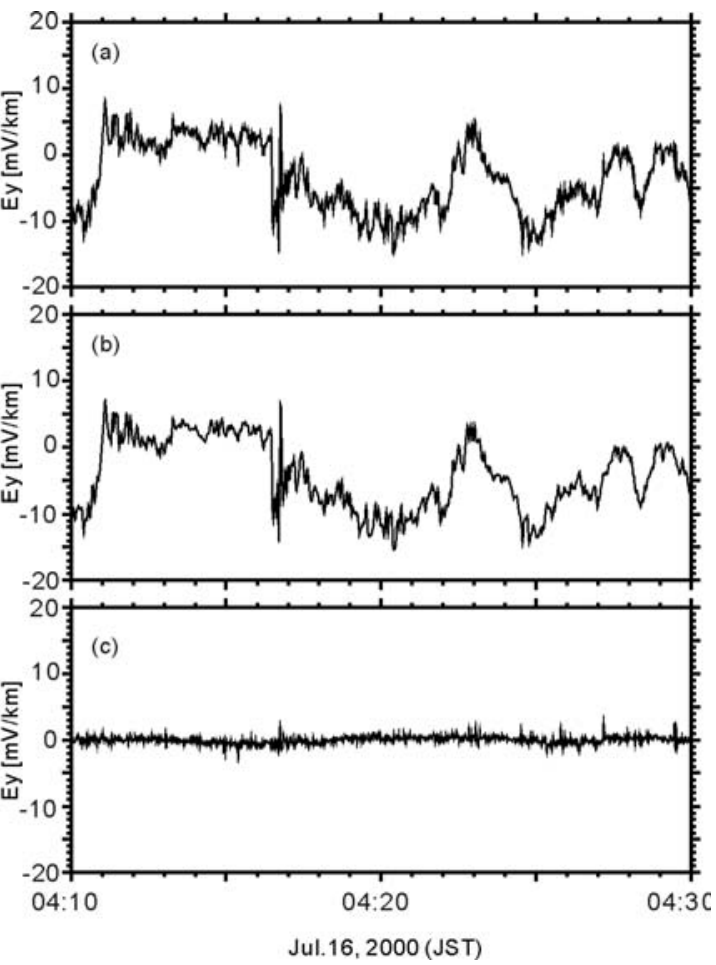

Fig. 4. The results of the reduction of inductive electric field associated with the external source field at KYS station for the interval of 04:10-04:30 on July 16, 2000 (JST); (1) $\mathrm{E}_{x}$ (NS) component, (2) $\mathrm{E}_{y}$ (EW) component. (a), (b), and (c) indicate the high-pass filtered $\left(\mathrm{T} \leq 940\right.$ s) original data at KYS, inductive electric field estimated from KAK by using $\mathbf{Z}_{i s}$ indicated in Fig.3, and residuals ((a)-(b)), respectively

geoelectromagnetic stations. There are two geomagnetic sensor arrays with inter-sensor distance of about $5 \mathrm{~km}$ in the Boso and Izu Peninsulas. Both regions are located in one of the most active seismic zones in Japan. Torsion-type magnetometers with three components manufactured by SPbF IZMIRAN are in operation ${ }^{(10)}$ and the horizontal electric field is measured in two perpendicular directions by pairs of electrodes, of which distance is about $50-$ $100 \mathrm{~m}$. All of equipments are synchronized by GPS clock. The sampling rate is $50 \mathrm{~Hz}$, but we use the data resampled down to 1 $\mathrm{Hz}$ in this study because the geomagnetic data observed at the Kakioka Magnetic Observatory (KAK) we use for reference is 1 Hz sampling. The location of KAK is also shown in Fig.1. In this paper, we apply the proposed method to the data obtained at KYS station in the Boso Peninsula. Fig.2 shows the observed magnetic data at KAK and KYS stations, and electric data at KYS in the 4 hours interval of 01:00- 05:00 (JST) on July 16, 2000, when the geomagnetic activity was extremely high ( $\mathrm{Kp}=9-)$.

Fig.3 shows the estimated $\mathbf{Z}_{i s}$ between the electric data at $\mathbf{K Y S}$ and the reference magnetic data at KAK, which are estimated for the midnight time (01:00-04:00, JST) on July 16, 2000. In order to obtain accurate $\mathbf{Z}_{i s}$, we calculated the multiple correlation functions of the linear system both in the time and scale domains, and take the average of subsections whose correlation was $\gamma^{2} \geq 0.9$. The transfer functions for period longer than 8 seconds seem to be well estimated. In Fig.3, open and solid circles indicate the real and imaginary part of $\mathbf{Z}_{i s}$, respectively.

Fig.4 shows the results of elimination of the inductive electric field associated with the global geomagnetic changes at KYS station. $E_{x}(\mathrm{NS})$ and $E_{y}(\mathrm{EW})$ components are showm in Figs.4 (1) and (2), respectively. In Fig.4, (a) illustrates the high-pass filtered
( $\leq 940$ s) original electric data at KYS and (b) represents the estimated inductive field at KYS by using the magnetic data at KAK. The waveform of Fig.4(b) closely resembles that of Fig.4(a), which suggests most of the electric field changes are correlated with the global geomagnetic variations. Fig.4(c) indicates the residual components (Fig.4 (a),(b)), showing that almost whole of the inductive field has been removed successfully, and the transient signals generated around KYS station appeared clearly.

\section{Verification of the Detectability of the Seismic Electric Signals}

As to the geoelectric potential difference data, SESs have been reported, the typical waveform being shown in Fig. ${ }^{(7)}$. The recognition of SES is usually checked by eye. Generally only a trend variation between electeric varaitions at a site and magnetic variations observed at some reference site is checked by eye. Therefore, the development of effective and easy methodology for reducting signals originated from external field variation is highly desired.

In order to evaluate the capability to extract the anomalous electric field changes associated with earthquakes, simple computation has been made. We simulate the SES type signals, which are assumed as the rectangular signals with $3 \mathrm{mV} / \mathrm{km}$ amplitude and various time durations from 10 to 300 seconds as shown in Fig.6. Each signal is marked by numbers in the panel. Then, these simulated SES signals are added to the observed electric data at KYS station, which is indicated in Fig.7(a). The data after imposing the simulated SES is described in Fig.7(b). The imposed SES signals are also illustrated in this panel. One 


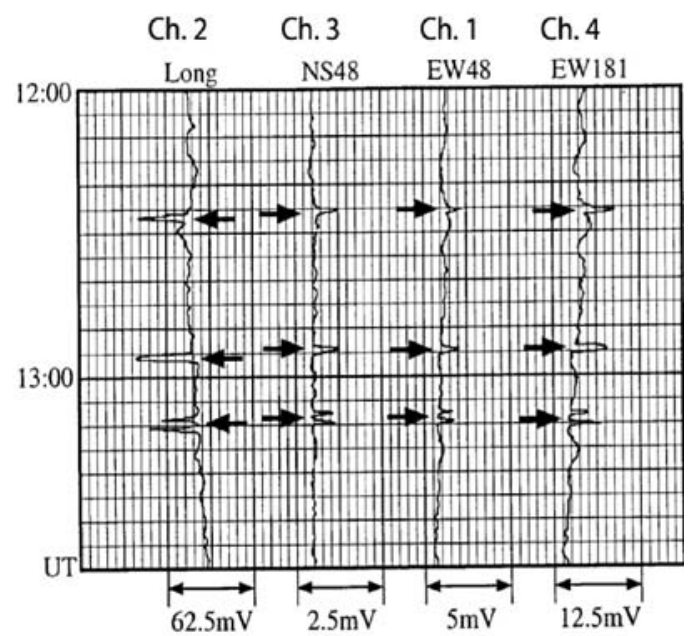

Fig. 5. Typical waveform of seismic electric signal (SES). After Varotsos et al. $(1991)^{(7)}$

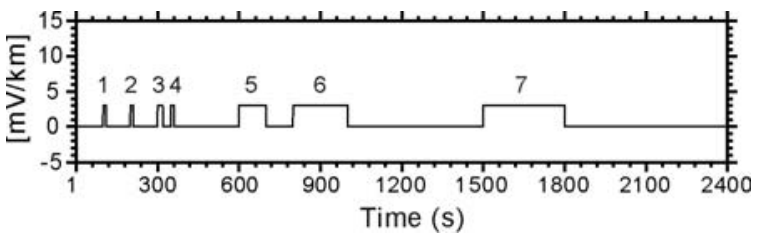

Fig. 6. The simulated rectangular waveform of "seismic electric signals (SES)" (Amplitude of them is $3[\mathrm{mV} / \mathrm{km}]$ and the width is (1) $10 \mathrm{~s},(2) 10 \mathrm{~s},(3) 20 \mathrm{~s}$, (4) $10 \mathrm{~s},(5) 100 \mathrm{~s}$, (6)200s, and (7)300s)
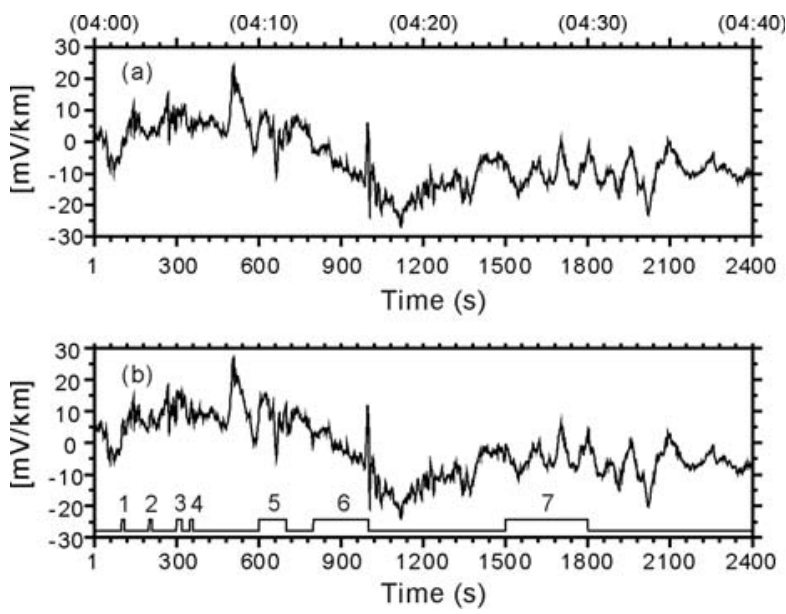

Fig. 7. Explanation of the procedure to make virtual geoelectric data with simulated SES; (a) The geoelectric field variations really observed at dipole 1 at KYS for the interval of 04:00-04:40 on July 16, 2000 (JST), (b)

The data with virtual SES as in Fig.6

cannot recognize the existence of such SES signals by eye. Fig. 8 shows the result of the signal discrimination with the proposed ISTF approach ( $\mathrm{T} \leq 940 \mathrm{~s})$. The numbers in the figure correspond to those in Figs.6 and 7. The inductive electric field related with the external source field were eliminated well, and the added simulated SES appeared clearly with keeping their characteristics of waveform.

Fig.9 illustrates the comparison of detection capability among different amplitude of imposed signals. The figures indicate that

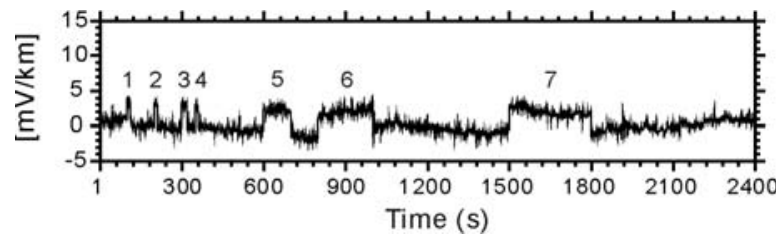

Fig. 8. The result for the detection of virtual SES. The numbers in the figure show the indices of SES in Figs. 6 and 7

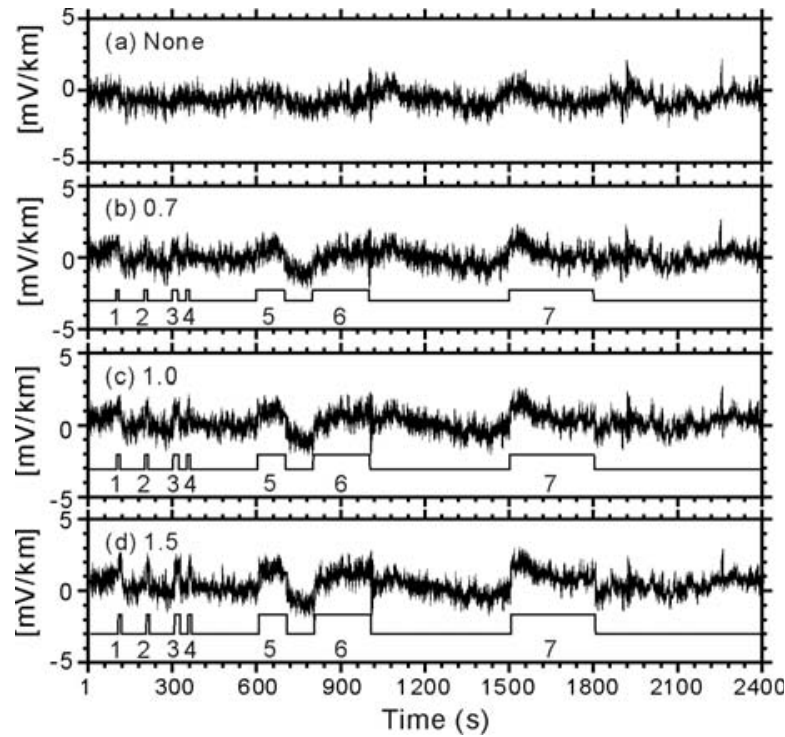

Fig. 9. The capability of the virtual SES separation against the background noise (The virtual SES are added to the really observed geoelectric variation as shown in Fig.7(a). The amplitude of virtual SES is a parameter in this computation. The results in the case of $0.0,0.7,1.0$, and $1.5 \mathrm{mV} / \mathrm{km}$ are described in (a), (b), (c) and (d). The imposed SES are also plotted)

lower limit of recognizable amplitude is about $1 \mathrm{mV} / \mathrm{km}$, which is comparable to the amplitude of the residual components which correspond to the background noise level at the observation site.

\section{Concluding Remarks}

The effctive method to eliminate signals originated from external magnetic variations in the frequency range of 0.001 to 1 $\mathrm{Hz}$ has been developed for geoelectric field data. It is based on the ISTF approach with the use of wavelet transform. It is important to estimate the interstation electromagnetic transfer functions $\mathbf{Z}_{\text {is }}$ with good accuracy. Therefore, we calculate $\mathbf{Z}_{i s}$ with high multicorrelation in Eq.(8). Using actual electromagnetic data observed at Kiyosumi station and reference magnetic data at Kakioka, we examine the effectiveness and performance of the proposed method for (1) reduction of inductive electric fields originated from external magnetic field and (2) detectability of SES.

The results are found to give good performance, which means that almost whole of the inductive fields are eliminated successfully and other signals are recognized with keeping their waveform. The detectability of simulated SES is high. Signals with amplitude similar to that of the background noise level (SNR $\geq 0 \mathrm{~dB}$ ) are detectable. In the near future, we will apply this method to actual cases to see if undetected possible 
electromagnetic changes associated with earthquakes may be discovered.

The residual $\Delta \mathbf{E}$ consists of regional and local signals, possibly including earthquake-related signals. On the other hand, the most intense signal in $\Delta \mathbf{E}$ is generally the leakage current from DC-driven trains in Japan. As the next step, we intend to establish a technique for discrimination of earthquake-related signals from electric field data contaminated with DC-train noise.

\section{Acknowledgement}

The authors wish to express their thanks to Kakioka Magnetic Observatory, Japan Meteorological Agency, for providing data. This work was partially supported by the JSPS Grants-in-Aid for Scientific Research \#13650477.

(Manuscript received Feb. 13, 2004, revised June 17, 2004)

\section{References}

(1) M. Hayakawa and O.A. Molchanov, Ed. : Seismo Electromagnetics, Lithosphere-Atmosphere-Ionosphere Coupling, p.477, TERRAPUB, Tokyo (2002)

(2) A.C. Fraser-Smith, A. Bernardi, P. R. McGill, M. E. Ladd, R. A. Helliwell, and O. G. Jr. Villard : "Low-frequency magnetic field measurements near the epicenter of the Ms 7.1 Loma Prieta earthquakes", Geophys. Res. Lett., Vol.17, pp.1465-1468 (1990)

(3) M. Hayakawa, R. Kawate, O. A. Molchanov, and K. Yumoto : "Result of ultra-low-frequency magnetic field measurements during the Guam earthquake of 8 August 1993", Geophys. Res. Lett., Vol.12, pp.241-244 (1996)

( 4 ) K. Hattori, Y. Akinaga, M. Hayakawa, K. Yumoto, T. Nagao, and S. Uyeda "ULF magnetic anomaly preceding the 1997 Kagoshima earthquakes", in Seismo Electroamgnetics : Lithosphere-Atmosphere-Ionosphere Coupling, M. Hayakawa and O. A. Molchanov, Ed., pp.19-28, TERRAPUB, Tokyo (2002)

( 5 ) S. Uyeda, M. Hayakawa, T. Nagao, O.A. Molchanov, K. Hattori, Y. Orihara, K. Gotoh, Y. Akinaga, and H. Tanaka : "Electric and magnetic phenomena observed before the volcano-seismic activity 2000 in the Izu islands region, Japan", Proc. US Nat. Acad. Sci., Vol.99, pp.7352-7355 (2002)

(6) P. Varotsos and K. Alexopoulos : "Physical properties of the variations of the electric field of the earth preceding earthquakes, I", Tectonophys., Vol.110, pp.73-98 (1984)

(7) P. Varotsos and M. Lazaridou : "Latest aspects of earthquake prediction in Greece based on seismic electric signals", Tectonophys., Vol.188, pp.321-347 (1991)

( 8 ) S. Uyeda, T. Nagao, Y. Orihara, T. Yamaguchi, and I. Takahashi : "Geoelectric potential changes: possible precursors to earthquakes in Japan", Proc. Natl. Acad. Sci. USA, Vol.97, pp.4561-4566 (2000)

(9) T. Nagao, Y. Orihara, T. Yamaguchi, I. Takahashi, K. Hattori, Y. Noda, K. Sayanagi, and S. Uyeda : "Co-seismic geoelectric potential changes observed in Japan", Geophys. Res. Lett., Vol.27, No.10, pp.1535-1538 (2000)

(10) K. Hattori, I. Takahashi, C. Yoshino, N. Isezaki, H. Iwasaki, M. Harada, K. Kawabata, E. Kopytenko, Y. Kopytenko, P. Maltsev, V. Korepanov, O.A Molchanov, M. Hayakawa, Y. Noda, T. Nagao, and S. Uyeda : "ULF geomagnetic field measurements in Japan and some recent results associated with Iwateken Nairiku Hokubu earthquake in 1998", Phys. Chem. Earth, Vol.29, pp.481-494 (2004)

(11) M. Harada, K. Hattori, and N. Isezaki : "Transfer function approach to signal discrimination of ULF geomagnetic data", Phys. Chem. Earth, Vol.29, pp.409-417 (2004)

(12) G. Chouliaras and T. M. Rasmussen : "The application of the magnetotelluric impedance tensor to earthquake prediction research in Greece", Tectnophys., Vol.152, pp.119-135 (1988)

(13) D. Hadjioannou, F. Vallianatos, K. Eftaxias, V. Hadjincotis, and K. Nomikos "Subtraction of the telluric inductive component from VAN measurements", Tectonophys., Vol.224, pp.113-124 (1993)

(14) L. Cagniard : "Basic theory of the magneto-telluric method of geophysical prospecting", Geophysics, Vol.112, pp.605-635 (1953)

(15) T. Rikitake and Y. Honkura : Solid Earth Geomagnetism, p.384, TERRAPUB, Tokyo (1985)

(16) L. Schmucker : "Anomalies of geomagnetic variations in the southwestern United States", Bull. Scripps Inst. Ocean., Univ. of California, Vol.13, pp.1-165 (1970)

(17) D. I. Gough and M. R. Ingham : "Interpretation methods for magnetometer arrays", Geophys. Space Phys., Vol.21, No.4, pp.805-827 (1983)
(18) G. D. Egbert and J. R. Booker : "Multivariate analysis of geomagnetic array data, 1. The response space", J. Geophys. Res., Vol.94, No.B10, pp.14227-14247 (1989)

(19) K. Vozoff: "The magnetotelluric method in the exploration of sedimentary basin", Geophysics, Vol.37, No.1, pp.98-141 (1972)

(20) I. Daubechies : Ten Lectures on Wavelets, p.357, Soc. Ind. and Appl. Math., Philadelphia (1992)

(21) J. Morlet : "Sampling theory and wave propagation", NATO ASI Series, 1 Issues in Acoustic Signal/Image Processing and Recognition, C.H. Chen, Ed., pp.233-261, Springer-Verlag (1983)

(22) Y-C. Zhang and K. V. Paulson : "Enhancement of signal-to-noise ratio in natural-source transient magnetotelluric data with wavelet transform", Pure Appl. Geophys., Vol.149, pp.405-419 (1997)

(23) H. Li : "Identification of coherent structure in turbulent shear flow with wavelet correlation analysis", J. Fluid Eng., Vol.120, pp.778-785 (1998)

(24) S. D. Meyers, B. G. Kelley, and J. J. O'Brien : "An introduction to wavelet analysis in oceanography and meteorology: With application to the dispersion of Yanai wave", Monthly Weather Rev., Vol.121, pp.2858-2866 (1993)

(25) C. Torrence and G. P. Compo : "A practical guide to wavelet analysis", Bull. Am. Meteor. Soc., Vol.79, No.1, pp.61-77 (1998)

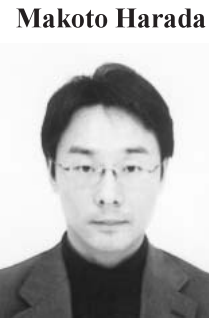

(Non-member) was born on April 22, 1973 in Japan. He received B.S., M.S., and Ph.D degrees from Chiba University, Japan in 1998, 2000, and 2004, respectively. He belongs to Earthquake Prediction Research Center, Tokai University since 2004. His research interests are in the areas of geophysics and signal processing with application to the continuous observation of the earthquake/volcanic activities. He is a member of the Society of Geomagnetism and Earth, Planetary and Space Sciences, the Society of Atmospheric Electricity of Japan, and the American Geophysical Union.

Katsumi Hattori (Member) was born on January 5, 1965 in Japan.

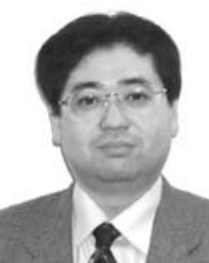
He received B.E., M.E., and D.E degrees from Nagoya University in 1987, 1989, and 1992, respectively. He worked at Toyama Prefectural University from 1992 to 1995 , National Gunma Institute of Technology from 1995 to 1997, RIKEN from 1997 to 2001 in Japan. He belongs to Marine Biosystems Research Center, Chiba University since 2001. He majors in radio science, signal processing, and geophysics. He is now making efforts to study and clarify earthquake-related electromagnetic phenomena, especially for realizing the short-term prediction. He is a member of the American Geophysical Union, URSI, Institute of Electronic Information Communications Engineers of Japan, the Institute of Electrical Engineers of Japan, the Society of Atmospheric Electricity of Japan, and the Society of Geomagnetism and Earth, Planetary and Space Sciences.

Nobuhiro Isezaki (Non-member) was born on November 20, 1943 in

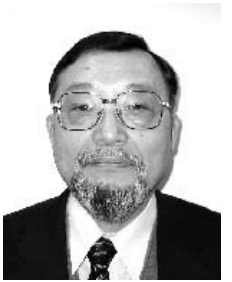
Japan. He received Ph.D degree in Geophysics from the University of Tokyo in 1971. He was with the Meteorological College, Japan Meteorological Agency as lecturer, and Kobe University as associate professor. Currently he is professor of Earth Sciences at Chiba University. His research interests are in the area of development of instruments and measurement of electromagnetic field on the earth's surface including the ocean bottom and the study of their time variation related to tectonic movements. $\mathrm{He}$ is a member of the Society of Geomagnetism and Earth, Planetary and Space Sciences, the Society of Seismology of Japan, the Geological Society of Japan, and the American Geophysical Union. 